Bull. Austral. Math. Soc.

VoL. 48 (1993) [41-46]

\title{
FREE COMMUTATIVE SEMIFIELDS
}

\section{B.J. GaRdNER}

In memory of Ottó Steinfeld

A description is obtained of the free semifields with both fundamental operations commutative.

A semiring $(A, \#, *)$, where $*$ is distributive over \#, is called a semifield if either $(A, *)$ is a group or \# has an identity (zero) $e$ and $\left(A \backslash\{e\},{ }^{*}\right)$ is a group. For many purposes there is no great loss of generality in restricting attention to the case where $(A, *)$ is a group (see $[4,5])$ and that is what we shall do here. Another reason for imposing this restriction is that the class of algebras so obtained (called proper semifields in [2]) is equational. Accordingly, we shall call an algebra $(A,+, \cdot)$ a semifield if $(A,+)$ is a group, $(A, \cdot)$ is a semigroup and + distributes over $\cdot$. (It is perhaps more common to assign names to the operations in the opposite way; we prefer to have the group operation called + because of ring-theoretic usage and because this is the natural notation in lattice ordered groups which constitute an important class of semifields.) We shall call a semifield commutative if both its operations are commutative. We shall obtain a description of the free commutative semifields and indicate some connections between these and free lattice ordered abelian groups.

Proposition 1. Let $X$ be a non-empty set, $F$ the free abelian group on $X$ (operation: + ), $S$ the free commutative semigroup on $F$ (operation: $\cdot$ ). Extend + is $S$ by defining

$$
a_{1} a_{2} \cdots a_{m}+b_{1} b_{2} \cdots b_{n}=\prod_{i=1}^{m} \prod_{j=1}^{n}\left(a_{i}+b_{j}\right) .
$$

Then $(S, \cdot,+)$ is a commutative semiring.

Proof: We have

$$
\begin{aligned}
\left(a_{1} a_{2}\right. & \left.\cdots a_{m}+b_{1} b_{2} \cdots b_{n}\right)+c_{1} c_{2} \cdots c_{k} \\
& =\Pi\left(a_{i}+b_{j}\right)+c_{1} \cdots c_{k}=\Pi\left(\left(a_{i}+b_{j}\right)+c_{r}\right) \\
& =\Pi\left(a_{i}+\left(b_{j}+c_{r}\right)\right)=a_{1} a_{2} \cdots a_{n}+\Pi\left(b_{j}+c_{r}\right) \\
& =a_{1} a_{2} \cdots a_{m}+\left(b_{1} b_{2} \cdots b_{n}+c_{1} c_{2} \cdots c_{k}\right),
\end{aligned}
$$

Received 20 June 1992

Copyright Clearance Centre, Inc. Serial-fee code: $0004-9729 / 93 \quad \$ A 2.00+0.00$. 
so + is associative, while

$$
\begin{aligned}
a_{1} a_{2} & \cdots a_{m}+b_{1} b_{2} \cdots b_{n}=\Pi\left(a_{i}+b_{j}\right)=\Pi\left(b_{j}+a_{i}\right) \\
& =b_{1} b_{2} \cdots b_{n}+a_{1} a_{2} \cdots a_{m}
\end{aligned}
$$

so + is commutative. Finally,

$$
\begin{gathered}
a_{1} a_{2} \cdots a_{m}+\left(b_{1} b_{2} \cdots b_{n} c_{1} c_{2} \cdots c_{k}\right)=\Pi\left(a_{i}+b_{j}\right) \Pi\left(a_{i}+c_{r}\right) \\
\left(a_{1} a_{2} \cdots a_{m}+b_{1} b_{2} \cdots b_{n}\right)\left(a_{1} a_{2} \cdots a_{m}+c_{1} c_{2} \cdots c_{k}\right),
\end{gathered}
$$

so + distributes over $\cdot$

Proposition 2. Addition in $S$ is cancellative.

PROOF: Let $a_{1} \cdots a_{m}+b_{1} \cdots b_{n}=a_{1} \cdots a_{m}+c_{1} \cdots c_{k}$, where all factors are in $F$. Then

$$
\prod_{i, j}\left(a_{i}+b_{j}\right)=\prod_{i, r}\left(a_{i}+c_{r}\right)
$$

As $F$ is torsion-free abelian, it carries a linear order, $<$, and we can assume that $a_{1} \leqslant a_{2} \leqslant \ldots \leqslant a_{m}, b_{1} \leqslant b_{2} \leqslant \ldots \leqslant b_{n}$ and $c_{1} \leqslant c_{2} \leqslant \ldots \leqslant c_{k}$. Since we have free generators, the same factors occur on each side of $\left(^{*}\right)$. Since clearly $a_{1}+b_{1} \leqslant a_{i}+b_{j}$ for all $i, j$ and $a_{1}+c_{1} \leqslant a_{i}+c_{r}$ for all $i, r$, we have $a_{1}+b_{1}=a_{1}+c_{1}$, so $b_{1}=c_{1}$. Thus we can re-write $\left({ }^{*}\right)$ as

$$
\prod_{i}\left(a_{i}+b_{1}\right) \prod_{j>1 ; i}\left(a_{i}+b_{j}\right)=\prod_{i}\left(a_{i}+b_{1}\right) \prod_{r>1 ; i}\left(a_{i}+c_{r}\right)
$$

and on cancelling, we get

$$
a_{1} \ldots a_{m}+b_{2} \ldots b_{n}=\prod_{j>1 ; i}\left(a_{i}+b_{j}\right)=\prod_{r>1 ; i}\left(a_{i}+c_{r}\right)=a_{1} \ldots a_{m}+c_{2} \ldots c_{k} .
$$

Repeating this argument, we see that the products $b_{1} \ldots b_{n}$ and $c_{1} \ldots c_{k}$ have the same factors with the same multiplicities.

As the semiring $(S, \cdot,+)$ has cancellative addition, we can form its semifield of differences $D(S)$. (We use this term rather than "semifield of quotients" as our distributive operation is called addition.) For details of this construction see Rédei [3, Theorem 93, p.160]. This is our free semifield.

ThEOREM. Let $X$ be a non-empty set, $F$ the free abelian group on $X, S$ the free commutative semigroup on $F$. Define $\prod_{i=1}^{m} a_{i}+\prod_{j=1}^{n} b_{j}=\prod_{i, j}\left(a_{i}+b_{j}\right)$ for all $a_{i}, b_{j} \in F$. 
This makes $(S, \cdot,+)$ a commutative semiring with cancellative addition and $D(S)$ is a free commutative semifield on $X$.

Proof: Let $A$ be a commutative semifield, $f: X \rightarrow A$ a function. Then there is a group homomorphism $f_{1}: F \rightarrow A$ defined by

$$
f_{1}\left(\sum n_{i} x_{i}\right)=\sum n_{i} f\left(x_{i}\right) .
$$

But then as $S$ is free on $F$, there is a semigroup homomorphism $f_{2}: S \rightarrow A$ given by

$$
f_{2}\left(a_{1}^{m_{1}} a_{2}^{m_{2}} \cdots a_{k}^{m_{k}}\right)=f_{1}\left(a_{1}\right)^{m_{1}} f_{1}\left(a_{2}\right)^{m_{2}} \cdots f_{1}\left(a_{k}\right)^{m_{k}},
$$

where $a_{1}, a_{2}, \cdots, a_{k} \in F$. Now consider $f_{2}$ in relation to + on $S$. If $a_{1}, a_{2}, \cdots, a_{m}$, $b_{1}, b_{2}, \cdots, b_{n} \in F$, then

$$
\begin{aligned}
& f_{2}\left(a_{1} a_{2} \cdots a_{m}+b_{1} b_{2} \cdots b_{n}\right) \\
& =f_{2}\left(\prod_{i, j}\left(a_{i}+b_{j}\right)\right)=\prod_{i, j} f_{1}\left(a_{i}+b_{j}\right)=\prod_{i, j}\left(f_{1}\left(a_{i}\right)+f_{1}\left(b_{j}\right)\right) \\
& =\prod_{j}\left(\prod_{i} f_{1}\left(a_{i}\right)+f_{1}\left(b_{j}\right)\right)=\prod_{i} f_{1}\left(a_{i}\right)+\prod_{j} f_{1}\left(b_{j}\right) \\
& =f_{2}\left(a_{1} a_{2} \cdots a_{m}\right)+f_{2}\left(b_{1} b_{2} \cdots b_{n}\right) .
\end{aligned}
$$

Thus $f_{2}$ preserves + . Finally, we define $f_{3}: D(S) \rightarrow A$ by setting

$$
f_{3}(u-v)=f_{2}(u)-f_{2}(v)
$$

for all $u, v \in S$. It is clear that $f_{3}$ is well-defined. For $u, v, w, z \in S$, we have

$$
\begin{aligned}
& f_{3}((u-v)+(w-z))=f_{3}((u+w)-(v+z))=f_{2}(u+w)-f_{2}(v+z) \\
& \quad=f_{2}(u)+f_{2}(w)-f_{2}(v)-f_{2}(z)=f_{2}(u)-f_{2}(v)+f_{2}(w)-f_{2}(z) \\
& \quad=f_{3}(u-v)+f_{3}(w-z)
\end{aligned}
$$

and

$$
\begin{aligned}
f_{3}((u-v)(w-z))=f_{3}((u+z)(v+w)-(v+z)) \\
\quad=f_{2}((u+z)(v+w))-f_{2}(v+z)=\left(f_{2}(u)+f_{2}(z)\right)\left(f_{2}(v)+f_{2}(w)\right)-f_{2}(v+z) \\
\quad=\left(f_{2}(u)+f_{2}(z)-f_{2}(v+z)\right)\left(f_{2}(v)+f_{2}(w)-f_{2}(v+z)\right) \\
\quad=\left(f_{2}(u)-f_{2}(v)\right)\left(f_{2}(w)-f_{2}(z)\right) \\
\quad=f_{3}(u-v) f_{3}(w-z) .
\end{aligned}
$$


Thus $f_{3}$ is a semifield homomorphism. For $x \in X$, we have

$$
\begin{aligned}
f_{3}(x+x-x) & =f_{2}(x+x)-f_{2}(x) \\
& =f_{2}(x)+f_{2}(x)-f_{2}(x) \\
& =f_{2}(x)=f_{1}(x)=f(x) .
\end{aligned}
$$

But $x+x-x$ is the copy of $x$ in $D(S)$. This completes the proof.

Let $\eta$ denote the minimum semilattice congruence on $(D(S), \cdot)$. Then $u \eta v$ if and only if each of $u, v$ divides or equals a power of the other [1, p.131]. Since, for example, $u w=v^{n}$ implies $(t+u)(t+w)=t+u w=t+v^{n}=(t+v)^{n}, \eta$ is in fact a semifield (semiring) congruence on $D(S)$, the minimum congruence for the variety defined by the identity $x^{2}=x$, that is the class of lattice-ordered abelian groups (see [4, Section 3 , Satz 1]). Thus $D(S) / \eta$ is a free lattice-ordered abelian group. Let us examine a bit more closely the relationship between the two free objects $D(S)$ and $D(S) / \eta$.

The original zero, 0 , of $F$ is an additive identity for $S:$ if $a_{1}, \ldots a_{m} \in F$, then

$$
a_{1} \ldots a_{m}+0=\left(a_{1}+0\right) \ldots\left(a_{m}+0\right)=a_{1} \ldots a_{m}
$$

We can thus identify each $u \in S$ with the corresponding $u-0$ in $D(S)$. Thus everything in $D(S)$ has the form $u-w$, where $u, w \in S$, so everything in $D(S) / \eta$ has the form $u \eta-w \eta$, where $u, w \in S$. If $u, v \in S$ and $u \eta v$, then either $u(r-s)=v^{n}$ or $u=v^{n}$ for some $r, x \in S, n \in \mathbb{Z}^{+}$. In the former case we have $(v+s)^{n}=v^{n}+s=u(r-s)+s=$ $(u+s)(r-s+s)=(u+s) r$ and in the latter, $(v+0)^{n}=u+0$. Thus there exists $s \in S$ such that $u+s$ divides or equals a power of $v+s$ in $S$. Similarly there exists $t \in S$ such that $v+t$ divides or equals a power of $u+t$ in $S$. But if $(v+s)^{n}=(u+s) r$ and $(u+t)^{m}=(v+t) q$, then $(v+s+t)^{n}=(v+s)^{n}+t=(u+s) r+t=(u+s+t)(r+t)$ and $(u+s+t)^{m}=(u+t)^{m}+s=(v+t) q+s=(v+s+t)(q+s)$ while if $(v+s)^{n}=$ $(u+s) r$ and $(u+t)^{m}=v+t$, then $(u+s+t)^{m}=(u+t)^{m}+s=v+s+t$, and so on. Thus in the above we can replace $s, t$ by $s+t$, that is assume $s=t$. We define the relation $\eta^{*}$ on $S$ as follows:

$u \eta^{*} v$ and only if there exists $s \in S$ such that each of $u+s, v+s$ divides or equals a power of the other in $S$.

This is the congruence induced on $S$ by $\eta$, so $D(S) / \eta=D\left(S / \eta^{*}\right)$. Suppose now $a_{1} \ldots a_{m}=u \eta^{*} v=b_{1} \ldots b_{n}$, where the factors are in $F$. Then there exist $c_{1}, \ldots, c_{k}, e_{1}, \ldots, e_{p} \in F$ and $\ell \in \mathbb{Z}^{+}$such that

or

$$
\begin{gathered}
\left(u+c_{1} \ldots c_{k}\right)\left(e_{1} \ldots e_{p}\right)=\left(v+c_{1} \ldots c_{k}\right)^{\ell} \\
\left(u+c_{1} \ldots c_{k}\right)=\left(v+c_{1} \ldots c_{k}\right)^{\ell}
\end{gathered}
$$


that is $\Pi\left(b_{g}+c_{h}\right)^{l}=\Pi\left(a_{i}+c_{j}\right) c_{1} \ldots e_{p}$ or $\Pi\left(a_{i}+c_{j}\right)$. Thus every $a_{i}+c_{j}$ is equal to some $b_{g}+c_{h}$. The converse holds also. On the other hand, if $\left\{a_{1}+c_{1}, \ldots, a_{1}+\right.$ $\left.c_{k}, \ldots, a_{m}+c_{k}\right\}=\left\{b_{1}+c_{1}, \ldots, b_{1}+c_{k}, \ldots, b_{n}+c_{k}\right\}$ then $u+c_{1} \ldots c_{k}\left(=\Pi\left(a_{i}+c_{j}\right)\right)$ divides a power of $v+c_{1} \ldots c_{k}\left(=\Pi\left(b_{g}+c_{h}\right)\right)$. This gives us another description of $S / \eta^{*}$.

Let $P_{f}(F)$ denote the set of finite non-empty subsets of $F$. Let + denote complex addition in $P_{f}(F)(A+B=\{a+b: a \in A, b \in B\})$. Then $\left(P_{f}(F),+, \cup\right)$ is a semiring. Let $\rho$ be defined on $P_{f}$ as follows:

$$
A \rho B \text { if and only if } A+C=B+C \text { for some } C \text {. }
$$

Then $S / \eta^{*} \cong P_{f}(F) / \rho ; D(S) / \eta$ is then the semifield of quotients of this. Note that $P_{f}(F)$ is the maximum semilattice (band) homomorphic image of $(F, \cdot)$ and $\rho$ is the minimum cancellative congruence on $\left(P_{f}(F),+\right)$. Each of these congruences is compatible with the "other" operation.

The congruence $\rho$ can be simply described when $|X|=1$, that is $F=\mathbb{Z}$. If $a, n \in \mathbb{Z}, n \geqslant 0$, then

$$
\begin{aligned}
\{a, a+1, \ldots, a+n\} & +\{a, a+1, \ldots, a+n\} \\
& =\{2 a, 2 a+1, \ldots, 2 a+n, 2 a+n+1, \ldots, 2 a+2 n\} \\
& =\{a, a+1, \ldots, a+n\}+\{a, a+n\},
\end{aligned}
$$

so $\{a, a+1, \ldots, a+n\} \rho\{a, a+n\}$. It now follows that $\{a, \ldots, a+i, \ldots, a+n\} \rho\{a, a+$ $n\}$ for any set of $a+i \in\{a+1, \ldots, a+n-1\}$ and thus that $A \rho\{\min (A), \max (A)\}$ for all $A \in P_{f}(\mathbb{Z})$. If $a, b, c, d \in \mathbb{Z}, a \leqslant b, c \leqslant d$ and $\{a, b\}+C=\{c, d\}+C$, then $a+\min (C)=\min (\{a, b\}+C)=\min (\{c, d\}+C)=c+\min (C)$ so $a=c$ and similarly $b=d$. Thus $\{a, b\} \rho\{c, d\}$ if and only if $\{a, b\}=\{c, d\}$ and we have

$$
P_{f}(\mathbb{Z}) / \rho \cong\{(a, b): a, b \in \mathbb{Z}, a \leqslant b\}
$$

where addition is componentwise and $(a, b)(c, d)=(\min \{a, c\}, \max \{b, d\})$.

The semifield of differences of this, that is the free lattice ordered group on one generator, has an additive group which is free of rank two - an old result of Birkhoff.

The congruence $\rho$ appears to be more difficult to describe for larger $X$.

\section{References}

[1] A.H. Clifford and G.B. Preston, The algebraic theory of semigroups Vol. 1 (American Mathematical Society, Providence, 1961). 
[2] H.C. Hutchins and H.J. Weinert, 'Homomorphisms and kernels of semifields', Period. Math. Hungar. 21 (1990), 113-152.

[3] L. Rédei, Algebra I (Pergamon Press, London, 1977).

[4] H.J. Weinert, 'Über Halbringe und Halbkörper I', Acta. Math. Acad. Sci. Hungar. 13 (1962), 365-378.

[5] H.J. Weinert, 'On 0-simple semirings, semigroup semirings, and two kinds of division semirings', Semigroup Fourm 28 (1984), 313-333.

Department of Mathematics The University of Tasmania GPO Box $252 \mathrm{C}$

Hobart, Tas. 7001

Australia 